\title{
0396 OBESITY AND THE RISK OF DEATH DURING TRAFFIC COLLISIONS
}

T M Rice*, M Zhu Correspondence: Department of Environmental Health Sciences, Safe Transportation Research and Education Center, University of California, Berkeley, 2614 Dwight Way \#7374, Berkeley, CA, 94122-7374, USA

10.1136/ip.2010.029215.396

Objective Obesity is also known to increase morbidity and decrease quality of life. While there is a large body of research on the health consequences of obesity, few studies have looked at how obesity affects traffic collision injury risk. The goal of this study was to estimate the effect of obesity on death risk during severe collisions.

Methods A matched-pair cohort study was conducted using data for years 1999-2008 from the Fatality Analysis Reporting System, a national fatal collision data system. Identified were 24936 pairs of adult drivers involved in two-vehicle collisions in which the vehicles were of similar type and size. Risk ratios for death were estimated for body mass index (BMI) quintiles using conditional Poisson regression.

Results Drivers in the lowest BMI quintile (BMI 12.9-21.5; RR 1.15, $p=0.08$ ) and the highest quintile (BMI 29.4-71.4; RR $1.28 \mathrm{p}=0.002$ ) appeared to have an elevated risk of death, compared with drivers in the lowest normal-weight quintile (BMI 21.5-23.9), adjusted for age, sex, seat belt use, air bag deployment, seat belt-air bag interaction and collision type.

Conclusions Underweight and obese vehicle occupants may be at higher risk of death than normal-weight occupants when involved in severe traffic collisions. The ability of passenger vehicles to protect obese occupants may have particular important public health implications, given the ongoing obesity epidemic in the USA. 